\title{
Erratum to: Spatial distribution of metals in top soils of Islamabad City, Pakistan
}

\author{
Syeda Maria Ali • Riffat Naseem Malik
}

Published online: 13 March 2010

(C) Springer Science+Business Media B.V. 2010

\section{Erratum to: Environ Monit Assess DOI 10.1007/s10661-010-1314-x}

The original version of this article unfortunately contained a mistake. In Table 1, the data in the second row (Detection limit) should be deleted. The correct table is given below.

Table 1 Specific instrument parameters and analysis of NIST-SRM-2709 and BCR-CRM-142R by AAS for each metal

\begin{tabular}{|c|c|c|c|c|c|c|c|c|c|c|c|}
\hline Elements & $\mathrm{Ca}$ & $\mathrm{Mg}$ & $\mathrm{Na}$ & $\mathrm{K}$ & $\mathrm{Fe}$ & $\mathrm{Cd}$ & $\mathrm{Co}$ & $\mathrm{Cu}$ & $\mathrm{Ni}$ & $\mathrm{Pb}$ & $\mathrm{Zn}$ \\
\hline$\lambda \max (\mathrm{nm})$ & 422.7 & 285.2 & 589 & 766.5 & 248.3 & 228.8 & 240.7 & 324.7 & 232 & 217 & 213.9 \\
\hline Flame type & $\mathrm{Air} / \mathrm{N}_{2} \mathrm{O}$ & Air & Air & Air & Air & Air & Air & Air & Air & Air & Air \\
\hline Limit of detection $\left(\mu \mathrm{g} \mathrm{g}^{-1}\right)$ & 0.053 & 0.01 & 0.05 & 0.10 & 0.053 & 0.010 & 0.092 & 0.029 & 0.03 & 0.03 & 0.007 \\
\hline Limit of quantification $\left(\mu \mathrm{g} \mathrm{g}^{-1}\right)$ & 0.15 & 0.03 & 0.16 & 0.32 & 0.16 & 0.03 & 0.27 & 0.08 & 0.11 & 0.11 & 0.02 \\
\hline Reference material & \multicolumn{6}{|c|}{ NIST-SRM-2709 (1992) (\%) } & \multicolumn{5}{|c|}{ BCR-CRM-142R (1989) $\left(\mu \mathrm{g} \mathrm{g}^{-1}\right)$} \\
\hline Certified value & 1.89 & 1.41 & 0.06 & 0.32 & 3.0 & 11.38 & 10.2 & 228.5 & 57.3 & 138.4 & 364.7 \\
\hline ed value & 1.71 & 1.22 & 0.063 & 0.41 & 2.7 & 15.4 & 9.8 & 225.2 & 70.5 & 110.6 & 274.6 \\
\hline Precision \% & 1.96 & 2.63 & 0.41 & 1.88 & 3.64 & 10.58 & 10.50 & 1.01 & 3.12 & 1.01 & 4.52 \\
\hline Recovery \% & 90.4 & 86.5 & 105 & 128 & 90 & 135.32 & 96 & 98.58 & 123.06 & 79.96 & 75.29 \\
\hline
\end{tabular}

The online version of the original article can be found at http://dx.doi.org/10.1007/s10661-010-1314-x.

S. M. Ali $(\varangle) \cdot$ R. N. Malik

Environmental Biology, Department of Plant Sciences,

Quaid-i-Azam University, Islamabad, Pakistan

e-mail: maria.ali01@yahoo.com 